\title{
Análise espaço-temporal da temperatura da superfície terrestre na Cidade de
}

\section{Marabá, Pará, Brasil}

\author{
Spatio-temporal analysis of the earth's surface temperature in Marabá City, Pará, Brazil \\ Análisis espacio-temporal de la temperatura superficial terrestre en la Ciudad de Marabá, Pará,
}

Brasil

Recebido: 01/06/2021 | Revisado: 07/06/2021 | Aceito: 14/06/2021 | Publicado: 27/06/2021

\author{
João Paulo Soares da Silva \\ ORCID: https://orcid.org/0000-0003-2124-0129 \\ Universidade do Estado do Pará, Brasil \\ E-mail: jp.22soares@gmail.com \\ Glauber Epifanio Loureiro \\ ORCID: https://orcid.org/0000-0002-0801-5296 \\ Universidade do Estado do Pará, Brasil \\ E-mail: epfanio@uepa.br \\ Italo de Sousa \\ ORCID: https://orcid.org/0000-0002-2212-4413 \\ Universidade do Estado do Pará, Brasil \\ E-mail: italo.uepa@hotmail.com
}

\begin{abstract}
Resumo
O desenvolvimento acelerado e desorganizado dos centros urbanos tem causado inúmeros impactos a esses ambientes, principalmente no que diz respeito aos aspectos climáticos das cidades. Aliado a diminuição de áreas vegetadas em detrimento do crescimento de locais construídos, o aumento das temperaturas urbanas tem como consequência a formação dos fenômenos de Ilhas de Calor. Neste sentido, o presente artigo objetiva analisar espaço-temporalmente a temperatura superficial terrestre da cidade de Marabá em um corte temporal dos anos de 1988 a 2018. A metodologia utilizada consistiu na coleta, análise e tratamento de imagens dos satélites Landsat 5 e Landsat 8, junto a dados matriciais de uso do solo da plataforma MapBiomas, realizando a elaboração de mapas, cálculos e índices que possibilitaram a identificação e definição das ilhas de calor urbana e a caracterização da dinâmica de utilização terrestre. Observou-se uma variação média das temperaturas máximas de $35,26 \%$, onde as ilhas de calor tiveram um aumento de formação principalmente nos núcleos São Félix e Zona de Expansão Nova Marabá, ao passo que as áreas vegetadas tiveram uma diminuição de $43,86 \%$, onde esses mesmos núcleos urbanos apresentavam maior troca de áreas de Formações Florestais por Infraestrutura Urbana. Confirmando assim o papel da vegetação na regulação da temperatura da superfície e a premissa de que as áreas impermeabilizadas ou com solo exposto são mais propensas à formação de ilhas de calor urbano.
\end{abstract}

Palavras-chave: Climatologia; Ilhas de Calor; Sensoriamento Remoto.

\begin{abstract}
The accelerated and disorganized development of urban centers has caused several impacts to these environments, especially with regard to the climatic aspects of cities. Combined with the reduction of vegetated areas to the detriment of the growth of built sites, the increase in urban temperatures has the consequence of the formation of the Heat Islands phenomena. Due to this, the present article aims to analyze space-time the terrestrial surface temperature of the city of Marabá in a temporal cut the months of July and August, from 1988 to 2018. The methodology used consisted of the collection, analysis and treatment of images from Landsat satellites 5 and 8 , composed with land use data from the MapBiomas platform, preparation of maps, calculations and indices that enabled the identification and definition of urban heat islands and the characterization of land use dynamics. An average variation of maximum temperatures of $35.26 \%$ was observed, where the heat islands had an increase in formation mainly in the São Félix and Zona de Expansão Nova Marabá, while the vegetated areas had a decrease of $43.86 \%$, where these same urban centers presented a greater exchange of areas of Forest Formations for Urban Infrastructure. Thus, confirming the role of vegetation in regulating the surface temperature and the premise that waterproofed areas or areas with exposed soil are more likely to form urban heat islands.
\end{abstract}

Keywords: Climatology; Heat Islands; Remote sensing. 


\begin{abstract}
Resumen
El desarrollo acelerado y desorganizado de los centros urbanos ha provocado numerosos impactos en estos entornos, principalmente en lo que respecta a los aspectos climáticos de las ciudades. Combinado con la reducción de áreas con vegetación en detrimento del crecimiento de los sitios construidos, el aumento de las temperaturas urbanas tiene la consecuencia de la formación de los fenómenos de las Islas de Calor. En este sentido, este artículo tiene como objetivo analizar espacio-tiempo la temperatura superficial terrestre de la ciudad de Marabá en un corte temporal de 1988 a 2018. La metodología utilizada consistió en la recolección, análisis y tratamiento de imágenes del Landsat 5 y Landsat 8. satélites, junto con datos de uso del suelo de la plataforma MapBiomas, realizando mapas, cálculos e índices que permitieron la identificación y definición de islas de calor urbano y la caracterización de la dinámica del uso del suelo. Se observó una variación promedio de temperaturas máximas de 35,26\%, donde las islas de calor tuvieron un aumento de formación principalmente en los núcleos São Félix y Zona de Expansão Nova Marabá, mientras que las áreas vegetadas tuvieron una disminución de 43,86\%, donde estos mismos centros urbanos presentó un mayor intercambio de áreas de Formaciones Forestales por Infraestructura Urbana. Confirmando así el papel de la vegetación en la regulación de la temperatura superficial y la premisa de que las áreas impermeables o con suelo expuesto son más propensas a la formación de islas de calor urbanas.
\end{abstract}

Palabras Clave: Climatología; Islas de calor; Detección remota.

\title{
1. Introdução
}

O estudo do clima está relacionado às características da atmosfera e às suas relações com o espaço geográfico e a sociedade. Assim, verifica-se a importância dos estudos de temperatura, visto que este aspecto afeta de maneira efetiva o meio socioambiental, por meio de alterações climáticas e sua influência na manutenção da vida das populações expostas a essa variante, como a formação de ilhas de calor e stress térmico (Cremonez et al., 2014).

Definido como um sistema que abrange o clima de um determinado espaço terrestre e sua urbanização o clima urbano é um dos aspectos ambientais mais afetados pelo abundante e gradativo crescimento das cidades (Oscar Junior; Brandão, 2015). Intensificado por um padrão de crescimento marcado por uma expansão horizontal, e de maneira a progredir sobre áreas verdes e um crescimento vertical, o avanço urbanístico exerce a partir dessas circunstâncias sua influência na climatologia das cidades (Almeida Junior et al., 2019) (Silva, et al., 2020).

Na mesma perspectiva, o comportamento térmico retrata o uso e ocupação terrestre e, no caso das cidades, reflete também o crescimento da mancha urbana. Essa relação se dá, pois, os materiais urbanos superficiais possuem padrões de reflectância, denominados como albedo, que favorecem a absorção ou reflectância da radiação e, com isso define a quantidade de calor emitido para a atmosfera (Sousa; Ferreira Junior, 2012).

Neste sentido, Fernandes (2015) infere que os estudos sobre o clima e principalmente os relacionados ao ambiente urbano apresentam os aspectos arquitetônicos que atuarão como retentores e refletores de calor, derivando dos materiais utilizados e os albedos, determinantes para a absorção e emissão de calor pela superfície. Alguns dos principais motivos do aquecimento das superfícies construídas são a impermeabilidade e a coloração desses materiais, que impedem a dissipação do calor do sol e materiais mais escuros absorvem e armazenam mais energia solar (Gartland, 2010).

A dinâmica terrestre se insere ainda mais nessa perspectiva, visto que aquelas superfícies mais claras tendem a refletir mais e reter menos calor, bem como superfícies com vegetação; já aquelas superfícies mais escuras e densas tendem a reter maior quantidade de calor, influenciando no acontecimento de fenômenos climáticos como as ilhas de calor urbano, que com o aumento das temperaturas nas áreas mais aglomeradas, vai interferir no conforto climático e na qualidade de vida das cidades (Fernandes, 2015) (Barboza et al., 2020)

Caracterizadas pela formação de núcleos com elevadas temperaturas cercados por locais menos quentes, as ilhas de calor são formadas em áreas urbanas visto que muitos materiais de construções comuns absorvem mais calor do sol em comparação a materiais naturais e a áreas rurais menos urbanizadas (Lucena, 2012). Para Ayoade (1998) os fenômenos de Ilhas de Calor são um dos aspectos mais importantes a serem observados no que diz respeito ao clima urbano, visto que são 
resultantes dos fatores antrópicos como uso e ocupação do solo, supressão da vegetação nativa, adensamento populacional, densidade e geometria das edificações. Logo a dinâmica territorial dos centros urbanos é um dos principais objetos de estudo a serem relacionados às pesquisas climáticas.

Neste sentido, apresenta-se a Temperatura Superficial Terrestre (TST), que está relacionada a detecção remota da radiação emitida pela superfície, assim sendo, uma temperatura aparente, ponderada em cadapixel da imagem (Borges; Zaidan, 2011). Segundo Fialho e Azevedo (2009), a utilização de dados de satélites determina a estimativa das características térmicas da cidade a partir da superfície dos telhados.

Utilizando imagens dos satélites Landsat 5 e Landsat 8 para obtenção de dados de temperatura da superfície, objetivase verificar as variações de temperatura e a identificação espacial de núcleos de calor ao longo de trinta anos na cidade de Marabá no Pará; junto a isso relacionar esses dados climáticos com o desenvolvimento territorial urbano e o uso do solo buscando alternativas para uma melhoria dos resultados encontrados. Este trabalho trata-se de uma pesquisa básica, de acordo com Pereira et al. (2018), com uma abordagem quanti-qualitativa. Conforme os objetivos essa pesquisa é considerada exploratória, e trata-se de um estudo de caso

\section{Materiais e Métodos}

\section{Área de Estudo}

A área de estudo abrange o perímetro urbano de Marabá, que de acordo com Marabá (2018) compreende os núcleos Marabá Pioneira, Cidade Nova, Nova Marabá, São Félix, Morada Nova, Zona de Expansão Urbana Nova Marabá, Zona de Expansão Urbana Cidade Nova, Distrito Industrial de Marabá - Etapas I e II e III. A Figura 1 mostra a divisão dos núcleos urbanos e a localização regional da cidade de Marabá.

Segundo Köppen (1928), Marabá de maneira regional tem seu clima definido como tropical quente e úmido (Awi), com precipitações anuais médias de $1800 \mathrm{~mm}$ e um período menos quente e mais chuvoso entre os meses de dezembro e abril e um período mais seco e de estiagem entre junho e outubro.

Nomeada como a cidade dos notáveis no auge mercantil da castanha, Marabá teve seu desenvolvimento alimentado por investimentos do governo federal na década de 1970 (Sposito et al, 2016) em seguida pelo Projeto Grande Carajás na década de 1980, que objetivava a exploração mineral em Carajás e fortaleceu Marabá como uma cidade referência na região, sofrendo um crescimento urbano e demográfico cada vez maior. Todo esse processo de urbanização acaba por resultar na substituição de áreas naturais, de vegetação nativa por objetos de construção, afetando a impermeabilidade do solo, substituindo os espaços por conta das atividades humanas, resultando no aumento das temperaturas locais em relação a áreas próximas podendo formar assim as ilhas de calor urbano (Rossato, 2011). 
Figura 1: Localização da cidade de Marabá, Pará.

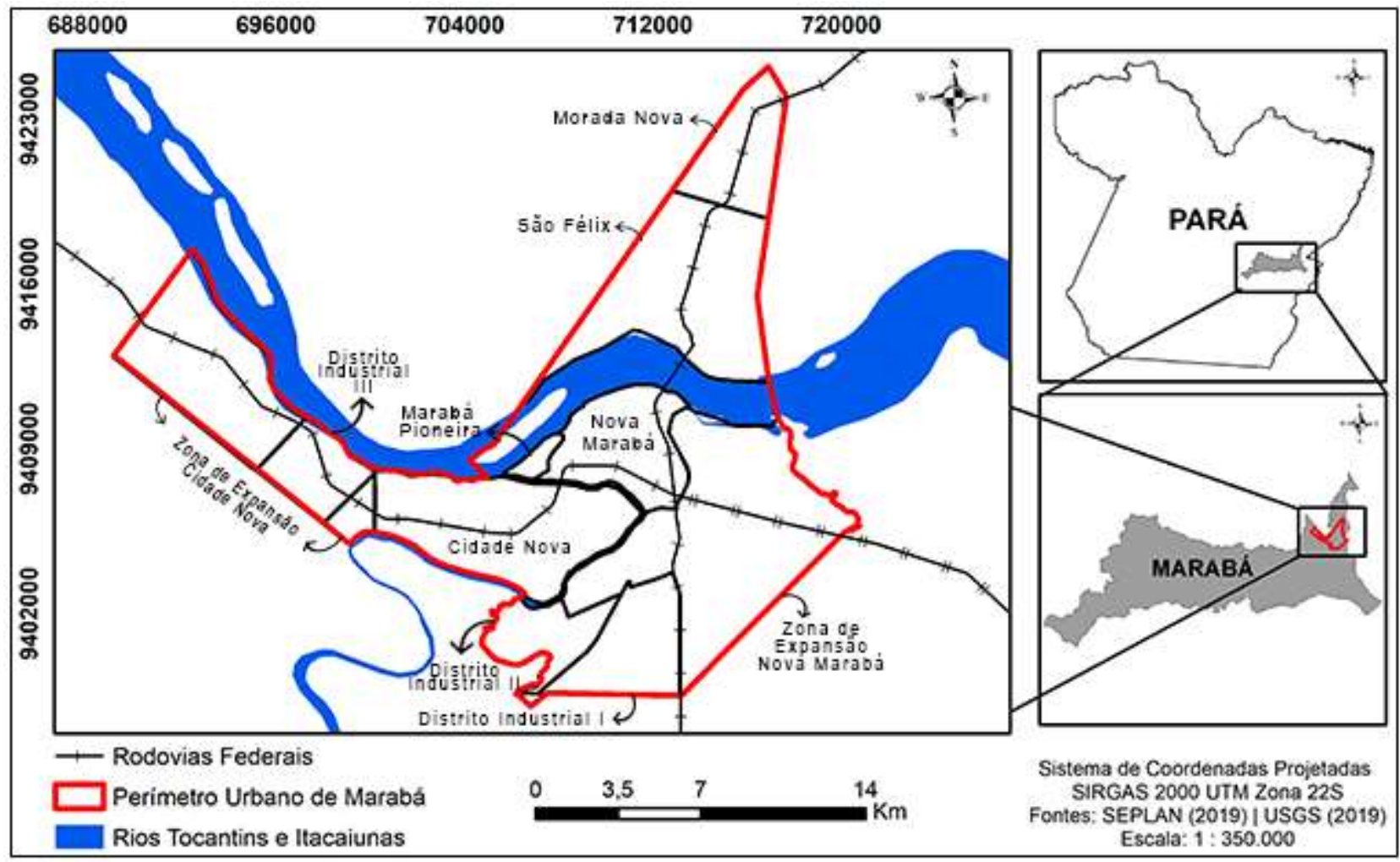

Fonte: Autores (2020).

\section{Elaboração dos mapas de TST}

A elaboração dos mapas de temperatura superficial terrestre (TST) se deu por meio do processamento de dados e metadados no software ArcGis (ESRI, 2017) com aplicação de ferramentas de reprojeção, recorte, bem como a calculadora raster para a realização das operações com equações de correção e geração dos mapas de TST.

A escolha dos anos de cada análise foi feita de acordo com a qualidade das imagens de maneira a evitar captação de traços de nuvens que pudessem interferir nos resultados de trocas espectrais entre a superfície e o satélite; e tentando manter uma ordem de 5 em 5 anos entre cada corte temporal. Inicialmente o mês de julho foi o escolhido para todos os anos, mas devido a problemas com a qualidade das imagens foram utilizadas cenas do mês de agosto nos anos de 1991 e 2001 conforme mostra a Tabela 1.

Com uma extensão territorial de 291,307 km², o perímetro urbano de Marabá está dentro de somente um quadrante ou órbitas/pontos da série Landsat; a partir disso foram adquiridas as imagens dos satélites Landsat 5 (1988 a 2011 ) e Landsat 8 (2016 e 2018) correspondentes à órbita/ponto 223/64. 
Tabela 1: Conjunto de metadados das imagens de satélite da série Landsat.

\begin{tabular}{|c|c|c|c|c|c|c|c|c|}
\hline $\begin{array}{c}\text { Satélite / } \\
\text { Banda }\end{array}$ & Data & Horário & $\mathbf{K}_{\mathbf{1}}$ & $\mathbf{K}_{2}$ & $Q_{c_{\max }}$ & $Q_{c_{\text {min }}}$ & $\begin{array}{c}L_{\max \lambda} \\
\left(W / m^{2} \cdot s r \cdot \mu m\right)\end{array}$ & $\begin{array}{c}L_{\min \lambda} \\
\left(W / m^{2} \cdot s r \cdot \mu m\right)\end{array}$ \\
\hline & $22 / 07 / 1988$ & $12: 55 \mathrm{~h}$ & & & & & & \\
\hline & 16/08/1991 & $12: 47 \mathrm{~h}$ & & & & & & \\
\hline Landsat- $5 \mathrm{TM}$ & 12/07/1996 & $12: 38 \mathrm{~h}$ & & & & & & \\
\hline / & $26 / 07 / 2001$ & $13: 04 \mathrm{~h}$ & 607.76 & 1260.56 & 255 & 1 & 15.303 & 1.238 \\
\hline \multirow[t]{2}{*}{ Banda 6} & $24 / 07 / 2006$ & $13: 17 \mathrm{~h}$ & & & & & & \\
\hline & 07/08/2011 & $13: 13 \mathrm{~h}$ & & & & & & \\
\hline $\begin{array}{c}\text { Landsat- } 8 \\
\text { OLI- TIRS / } \\
\text { Banda } 10\end{array}$ & $\begin{array}{l}03 / 07 / 2016 \\
09 / 07 / 2018\end{array}$ & $\begin{array}{l}13: 24 \mathrm{~h} \\
13: 23 \mathrm{~h}\end{array}$ & 774.8853 & 1321.07 & 65535 & 1 & 22.00180 & 0.10033 \\
\hline
\end{tabular}

Fonte: adaptado de USGS (2019).

Para extrair os dados referentes à temperatura superficial a partir da banda 6 das imagens Landsat-5 TM é necessário converter os números digitais (DN), valor do pixel, para valores TST; por meio da Equação 1 é possível realizar a conversão dos números digitais para radiância espectral $\left(\mathrm{L}_{\lambda}\right)$. Essa equação foi introduzida pela NASA e tem sido bastante utilizada em diversos estudos sobre ilhas de calor urbanas (Qin et al, 2001) (Esri, 2017).

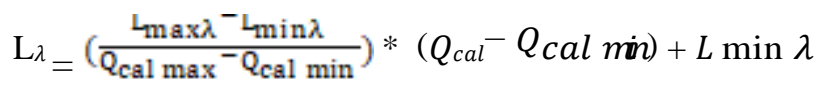

onde:

$L_{\lambda}=$ Radiância espectral $\left(W / m^{2} \cdot s r \cdot \mu m\right)$

$Q_{\text {cal }}=$ Valor quantizado e calibrado do pixel em nível de cinza (DN)

$Q_{\text {cal min }}=$ Valor mínimo do pixel em níveis de cinza $(\mathrm{DN}=1)$

$Q_{\text {cal } \max }=$ Valor mínimo do pixel em níveis de cinza $(\mathrm{DN}=255)$

$L_{\min \lambda}=$ Radiância espectral mínima $\left(1.238 \mathrm{~W} / \mathrm{m}^{2} \cdot \mathrm{sr} \cdot \mu \mathrm{m}\right)$

$L_{\max \lambda}=$ Radiância espectral máxima $\left(15.303 \mathrm{~W} / \mathrm{m}^{2} \cdot \mathrm{sr} \cdot \mu \mathrm{m}\right)$

Uma vez que o valor de radiância espectral ( $\mathrm{L} \lambda$ ) fora conhecido, por conseguinte é utilizada essa variável para calcular a Temperatura Superficial Terrestre, a Equação 2 é responsável por determinar os valores de TST em Kelvin ( $\left.{ }^{\circ} \mathrm{K}\right)(\mathrm{Q} i n$ et al, 2001) (Esri, 2017).

$$
T k=\frac{\mathrm{K}_{2}}{\ln \left(\frac{K_{1}}{[-\pi}+1\right)}
$$

onde:

$\mathrm{T}_{\mathrm{k}}=$ Temperatura com correção atmosférica $\left({ }^{\circ} \mathrm{K}\right)$

$\mathrm{K}_{1}=$ Constante de calibração $1(\mathrm{~W} / \mathrm{m} 2 \mathrm{sr} \mu \mathrm{m})$

$\mathrm{K}_{2}=$ Constante de calibração $2(\mathrm{~K})$

$\mathrm{L}_{\lambda}=$ Radiância de um alvo negro de temperatura cinética $(\mathrm{W} / \mathrm{m} 2 \cdot \mathrm{sr} \cdot \mu \mathrm{m})$

Com o valor das temperaturas em Kelvin $\left({ }^{\circ} \mathrm{K}\right)$, elas foram convertidas para graus Celsius $\left({ }^{\circ} \mathrm{C}\right)$ com a subtração do valor da temperatura do ponto de congelamento da água ao nível do mar, que equivale a $273,15^{\circ} \mathrm{K}$ (Esri, 2017).

Para o cálculo da temperatura de superfície das imagens do satélite Landsat 8, inicialmente foi aplicada a Equação 1 
nas imagens para obtenção da radiância espectral ( $\mathrm{L} \lambda$ ), adequando os valores das constantes presentes na equação com os metadados das imagens Landsat 8.

Por conseguinte, foram obtidos os valores de transmissividade atmosférica, radiância emitida e recebida pela superfície, conforme dados da Tabela 2; para que em seguida fosse efetuado o cálculo da radiância $\left(\mathrm{L}_{T}\right)$ através da Equação 3 (Barsi et al., 2003).

$$
\mathrm{L} T=\frac{\mathrm{L}_{\lambda}-\mathrm{Lu}-(1-\varepsilon) \mathrm{L}_{\mathrm{d}}}{\tau \varepsilon}
$$

onde:

$L_{T}=$ Radiância de um alvo negro de temperatura cinética $(\mathrm{W} / \mathrm{m} 2 \cdot \mathrm{sr} \cdot \mu \mathrm{m})$

$\mathrm{L}_{\lambda}=$ Radiância espectral $(W / m 2 \cdot s r \cdot \mu m)$

$\tau=$ Transmissividade da atmosfera

$\varepsilon=$ Emissividade da superfície

$L u=$ Radiância emitida pela superfície $(\mathrm{W} / \mathrm{m} 2 \cdot \mathrm{sr} \cdot \mu \mathrm{m})$

$L d=$ Radiância recebida pela superfície $(\mathrm{W} / \mathrm{m} 2 \cdot \mathrm{sr} \cdot \mu \mathrm{m})$

Tabela 2: Dados de entrada e saída para a correção atmosférica das imagens Landsat 8.

\begin{tabular}{cccc}
\hline Dados & Data da imagem & $\mathbf{0 3 / 0 7 / 2 0 1 6}$ & $\mathbf{0 9 / 0 7 / 2 0 1 6}$ \\
\hline \multirow{2}{*}{ Entrada } & Latitude/Longitude & $-4.738 /-49.841$ & $-4.738 /-49.844$ \\
& Altitude & $0.084 \mathrm{~km}$ & $0.084 \mathrm{~km}$ \\
& Pressão & $1000 \mathrm{mb}$ & $1000 \mathrm{mb}$ \\
& Temperatura & $29.38^{\circ} \mathrm{C}$ & $29.20^{\circ} \mathrm{C}$ \\
Saída & Umidade Relativa & $55.5 \%$ & $55.5 \%$ \\
& Radiância emitida pela superfície & $3.29 \mathrm{~W} / \mathrm{m}^{2} \cdot \mathrm{sr} \cdot \mu \mathrm{m}$ & $2.69 \mathrm{~W} / \mathrm{m}^{2} \cdot \mathrm{sr} \cdot \mu \mathrm{m}$ \\
& Radiância recebida pela superfície & $5.09 \mathrm{~W} / \mathrm{m}^{2} \cdot \mathrm{sr} \cdot \mu \mathrm{m}$ & $4.28 \mathrm{~W} / \mathrm{m}^{2} \cdot \mathrm{sr} \cdot \mu \mathrm{m}$ \\
\hline
\end{tabular}

Fonte: (NASA, 2019); (USGS, 2019)

A partir da obtenção da radiância com a correção $\left(\mathrm{L}_{T}\right)$, ela foi aplicada na Equação 2 para obtenção dos dados de temperatura em ${ }^{\circ} \mathrm{K}$, e repetido o processo de conversão de Kelvin para Celsius. Cabe citar que se optou em utilizar o valor de emissividade de 0,92, visto que se refere ao valor médio das superfícies urbanas (Nichol, 1994).

A partir disso as imagens de temperatura de superfície foram classificadas e, por conseguinte realizada a aplicação de um design às imagens resultantes, com o intuito de melhor identificar o comportamento da temperatura dos alvos nas imagens e mesclaraos mapas de uso do solo (Pires; Ferreira Junior, 2015).

\section{Elaboração dos Mapas de Uso e Ocupação do Solo}

Foram coletados na plataforma MapBiomas os dados de uso e ocupação do solo do município de Marabá em forma matricial e posteriormente submetidos ao processamento de classificação a partir dos códigos de legenda para os valores de pixels, onde cada valor representa um tipo de uso do solo (Esri, 2017; Projeto MAPBIOMAS, 2019). 
Em seguida foram realizadas as colorações e rotulagens de cada classe de pixels, deixando mais fácil a compreensão e distinção de cada tipo de formação natural ou não; junto a isso foram utilizadas ferramentas SIG como a calculadora de área a partir da tabela de atributos para calcular a área que cada tipo de uso ocupava dentro da cidade de Marabá (Esri, 2017).

Para uma melhor demonstração da metodologia elaborada, foram coletados dados de temperatura, evapotranspiração diária e precipitação diária da região em estudo registrados pela Estação 82562 do Instituto Nacional de Meteorologia (INMET) localizada na cidade de Marabá, de modo que a partir da comparação dos dados de TST obtidos através das imagens Landsat com os das estações sejam verificadas as diferenças ou semelhanças encontradas nas duas séries de dados.

\section{Resultados e Discussões}

\section{Análise para 1988 e 1991}

O ano de 1988 demonstrou os seguintes dados: temperatura mínima de $22,37^{\circ} \mathrm{C}$, máxima de $33,26{ }^{\circ} \mathrm{C}$ e uma média de $26,81{ }^{\circ} \mathrm{C}$; já em 1991 verificou-se uma diminuição da temperatura mínima para $18,38^{\circ} \mathrm{C}$, a elevação da máxima para $37,22^{\circ} \mathrm{C}$, e uma média de $27,80^{\circ} \mathrm{C}$.

A Figura 2 mostra nesse primeiro corte temporal que no ano de 1988 a área correspondente pelo perímetro urbano de Marabá apresentava 160,61 km² de Formações Florestais, uma área de 81,86 km² de Pastagens e Outras Formações, e 22,24 km² de Infraestrutura Urbana; já em 1991 apresentava áreas de 128,35 km², 95,07 km² e 27,25 km² respectivamente.

Figura 2: Mapa de uso do solo e temperatura da superfície da cidade de Marabá em 07/22/1988 e 08/16/1991.

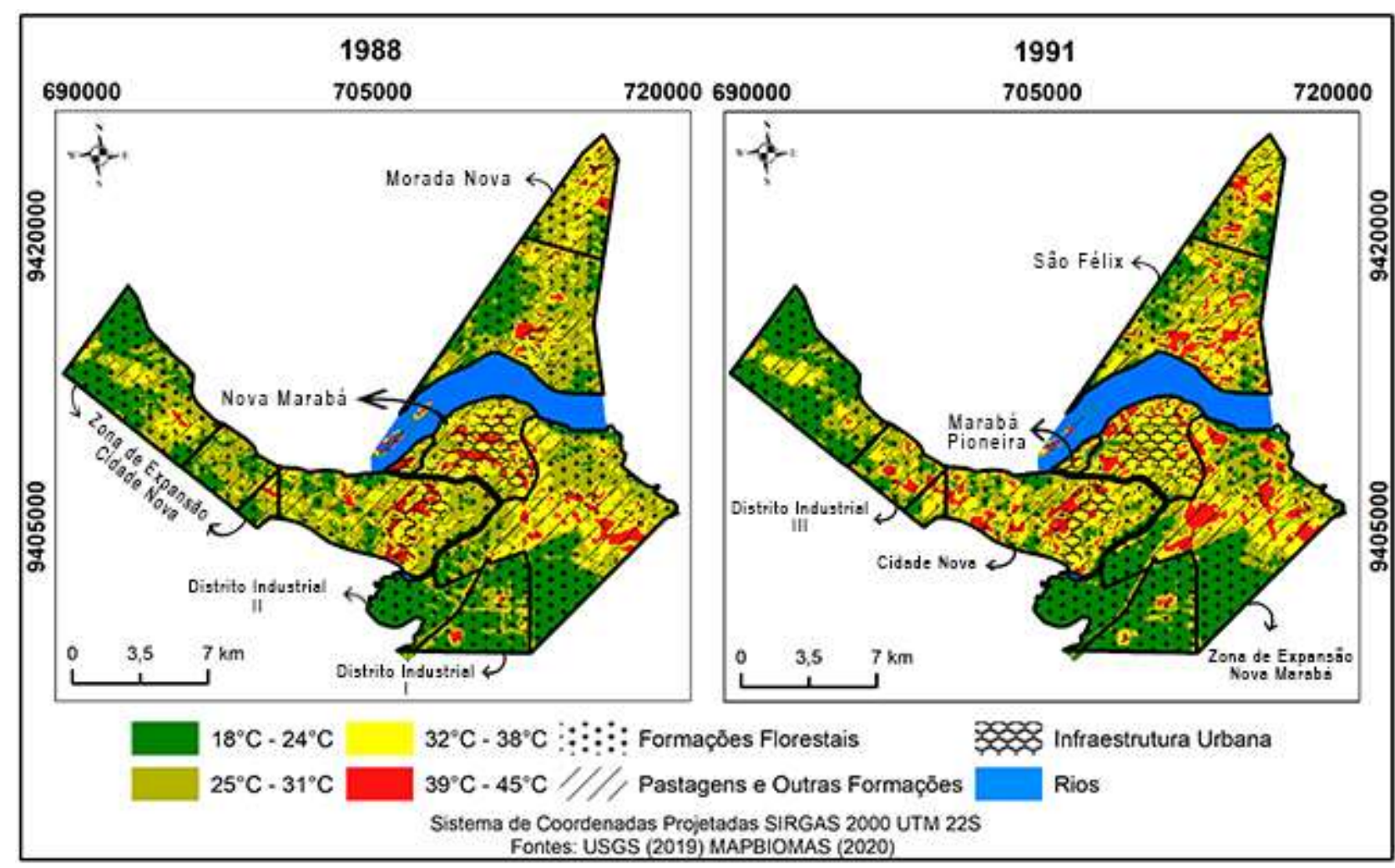

Fonte: Autores (2020).

Verificou-se que em 1988 os núcleos Cidade Nova, Nova Marabá foram os que apresentaram maiores formaçãoes de ilhas de calor, bairros esses recém-criados nessa época, visto que a cheia dos Rios Tocantins e Itacaiúnas fez com que o governo municipal criasse áreas habitacionais mais distantes dos rios. Já em 1991 os núcleos Zona de Expansão Nova Marabá, 
Cidade Nova e São Félix apresentaram as maiores formações de ilhas de calor e mais pontos com temperaturas entre $39^{\circ} \mathrm{C}$ e $45^{\circ} \mathrm{C}$.

Em análise dos mapa de utilização terrestre, verifica-se uma diminuição da área vegetada em defronte a um crescimento de construções e ocupações urbanas, alia-se a esse fato o aumento populacional que a cidade sofreu, na época com 102.435 habitantes segundo o censo do IBGE daquele ano.

Neste sentido Lima e Leão (2015) informam que no início da década de 1990, o núcleo São Félix recebeu ocupações irregulares na margem leste da PA-150 hoje BR-222, originando o atual bairro São Félix III, confirma-se essa tese de maneira que no mapa de 1988 existe uma mancha verde com coloração da classe de Formação Campestre, que em 1991 já é compreendida como Infraestrutura Urbana.

\section{Análise para 1996 e 2001}

A TST em 1996 apresentou valores mínimos de $20,17^{\circ} \mathrm{C}$, uma máxima de $30,42^{\circ} \mathrm{C}$ e média de $25,29^{\circ} \mathrm{C}$; na observação seguinte em 1991 a temperatura mínima era de $21,06^{\circ} \mathrm{C}$, máxima de $32,05^{\circ} \mathrm{C}$ e média de $26,55^{\circ} \mathrm{C}$, verificando assim um aumento gradativo ao passar dos anos verificados. A Figura 3 mostra que no que tange o emprego do solo, em 1996 verificou-se que 138,93 km² correspondiam por Formações Florestais, 91,28 km² por Pastagens e Outras Formações e, 29,67 km² por Infraestrutura Urbana; já em 2001 apresentou valores de 129,86 km², 109,72 km² e, 29,78 km² respectivamente.

Figura 3: Mapa de uso do solo e temperatura da superfície da cidade de Marabá em 12/07/1996 e 26/07/2001.

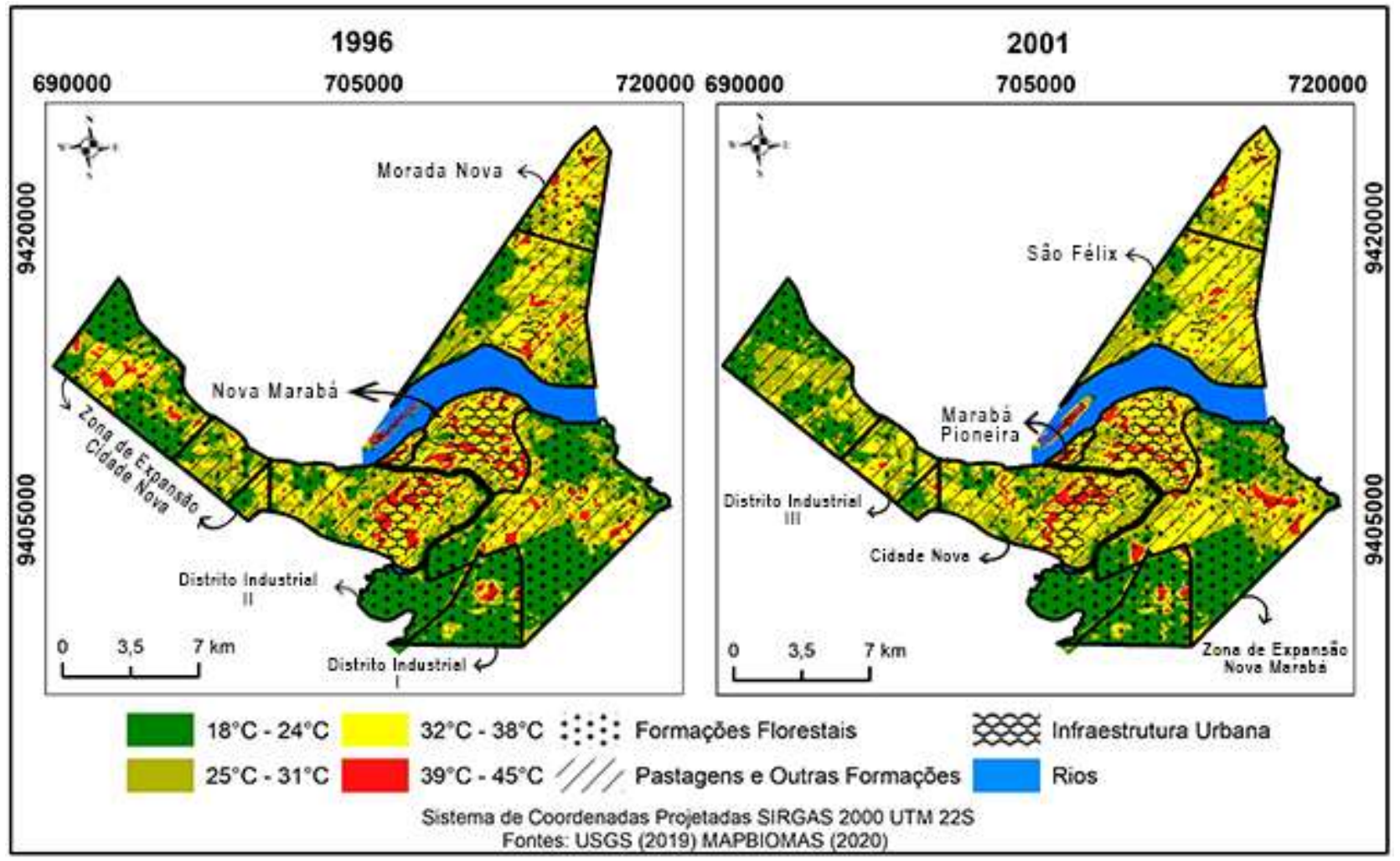

Fonte: Autores (2020).

Em 1996 os valores de TST apresentaram uma queda em relação a análise anterior, acompanhado do aumento das áreas de Formações Florestais em detrimento das outras ocupações visualizadas neste ano, esse fato pode ser associado ao aumento da quantidade de áreas de Formações Florestais; visto que que através do consumo de gás carbônico $\left(\mathrm{CO}_{2}\right)$ e produção 
de Oxigênio $\left(\mathrm{O}_{2}\right)$, as plantas atuam na melhoria da qualidade do ar, sendo um fator importante para os níveis de temperatura encontrados em qualquer ambiente (souza filho et al., 2006; velho, 2014).

Ao analisar a distribuição das ilhas de calor dentro do perímetro urbano, verifica- se poucas áreas com a classe de maior temperatura, entretanto nos Núcleos São Félix e Morada Nova percebe-se um aumento dos pontos com temperaturas máximas considerando o ano anterior. E a continuidade das maiores temperaturas nos núcleos Nova Marabá e Cidade Nova.

Colidente a essa constatação observa-se o aumento das áreas de Pastagem e Infraestrutura Urbana na disposição do solo da cidade, nesta perspectiva Ramos et al. (2016) infere que a crescente urbanização a partir da substituição da vegetação pela área urbanizada causa mudanças irreparáveis no ambiente, como alterações no microclima e desconforto térmico.

\section{Análise para 2006 e 2011}

A Temperatura Superficial Terrestre no ano de 2006 contava com mínima de $23,68^{\circ} \mathrm{C}$, máxima de $39,55^{\circ} \mathrm{C}$ e média de $31,61^{\circ} \mathrm{C}$, um aumento significativo em relação ao ano de 2001, além disso o ano de 2011 apresentou temperaturas da seguinte maneira: mínima de $24,64{ }^{\circ} \mathrm{C}$, máxima de $40,05^{\circ} \mathrm{C}$ e média de $32,34^{\circ} \mathrm{C}$. A Figura 4 explicita que em relação às interações da superfície urbana de Marabá, as áreas de Formações Florestais apresentavam 118,66 km² em 2006 e 127,71 km² em 2011; as de Pastagens e Outras Formações ocupavam 116,85 km² em 2006 e 99,18 km² em 2011; já em 200633,36 km² eram ocupados por Infraestrutura Urbana e 38,47 km² em 2011.

Figure 4: Mapa de uso do solo e temperatura da superfície da cidade de Marabá em 24/07/2006 e 07/08/2011.

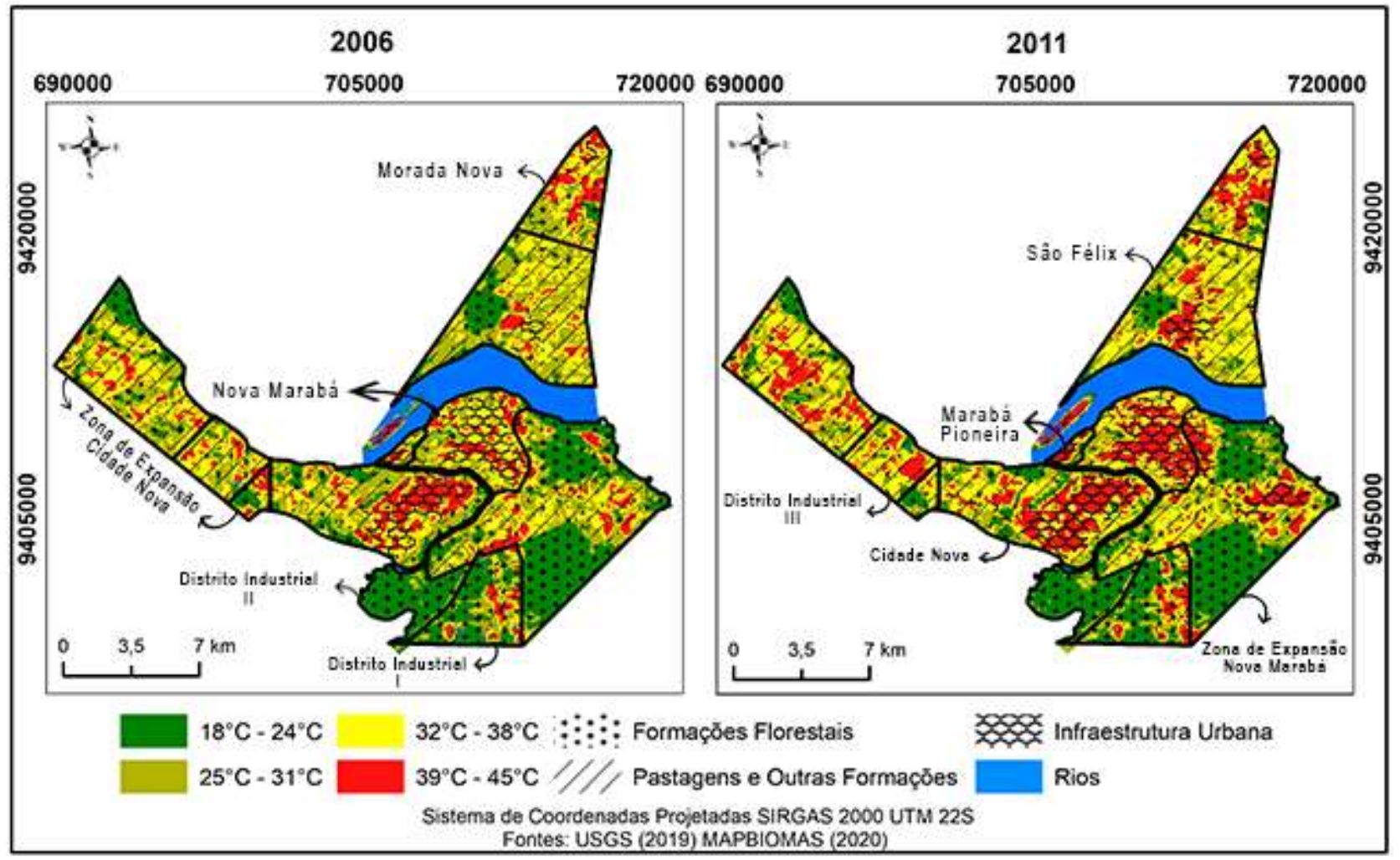

Fonte: Autores (2020).

Em comparação aos anos anteriores, observa-se um aumento dos valores de temperatura, sejam eles mínimos ou máximos; aliado a isso verifica-se nesse ano uma diminuição de cerca de $11 \mathrm{~km}^{2}$ de áreas de Formações Florestais enquanto constata-se um aumento das áreas de Pastagem e Infraestrutura Urbana. Nos locais da cidade de Marabá compostos por áreas 
de pastagens foram verificadas temperaturas dentro das duas maiores classes $\left(32^{\circ} \mathrm{C}\right.$ a $38^{\circ} \mathrm{C}$ e $\left.39^{\circ} \mathrm{C} \mathrm{a} 45^{\circ} \mathrm{C}\right)$ apesar de conter vegetação, pois segundo Primavesi et al (2007), quando as folhas secam, a reflectância captada é maior, resultando em maiores valores de temperatura na superfície.

O ano de 2011 mostrou os efeitos do processo de expansão urbana organizada da cidade que vinha acontecendo desde 2009, como a implantação de loteamentos nos núcleos Zona de Expansão Nova Marabá e Cidade Nova (Cidade Jardim, Delta Park e Mirante do Vale) e a instalação de quatro empreendimentos do Programa Minha Casa Minha Vida (PMCMV) nos núcleos São Félix e Morada Nova (Lima; Leão, 2015), sendo esses núcleos uns dos com maiores formações de ilhas de calor em 2006 e 2011, juntamente com os núcleos Cidade Nova e Nova Marabá com ilhas já consolidadas.

Esses fatores arquitetônicos e territoriais influenciam a TST de maneira que com a construção dos empreendimentos PMCMV, a construção civil e o mercado imobiliário de Marabá expandiram de maneira acentuada. Assim, o albedo dessas superfícies tomadas por coberturas de concreto ou telhas normais resultou níveis maiores de reflectância, originando assim maiores valores de temperatura.

\section{Análise para 2016 e 2018}

No ano de 2016, a temperatura mínima observada para a delimitação urbana de Marabá foi de $28,15^{\circ} \mathrm{C}$, com máxima de $42,16^{\circ} \mathrm{C}$ e média de $35,15^{\circ} \mathrm{C}$, resultados semelhantes foram também encontrados nos estudos de Silva et al. (2020); o ano de 2018 apresentou temperatura mínima de $29,27^{\circ} \mathrm{C}$, máxima de $44,99^{\circ} \mathrm{C}$ e média de $37,13^{\circ} \mathrm{C}$, assim demonstrado na Figura 5 .

Figura 5: Mapa de uso do solo e temperatura da superfície da cidade de Marabá em 03/07/2016 e 09/07/2018.

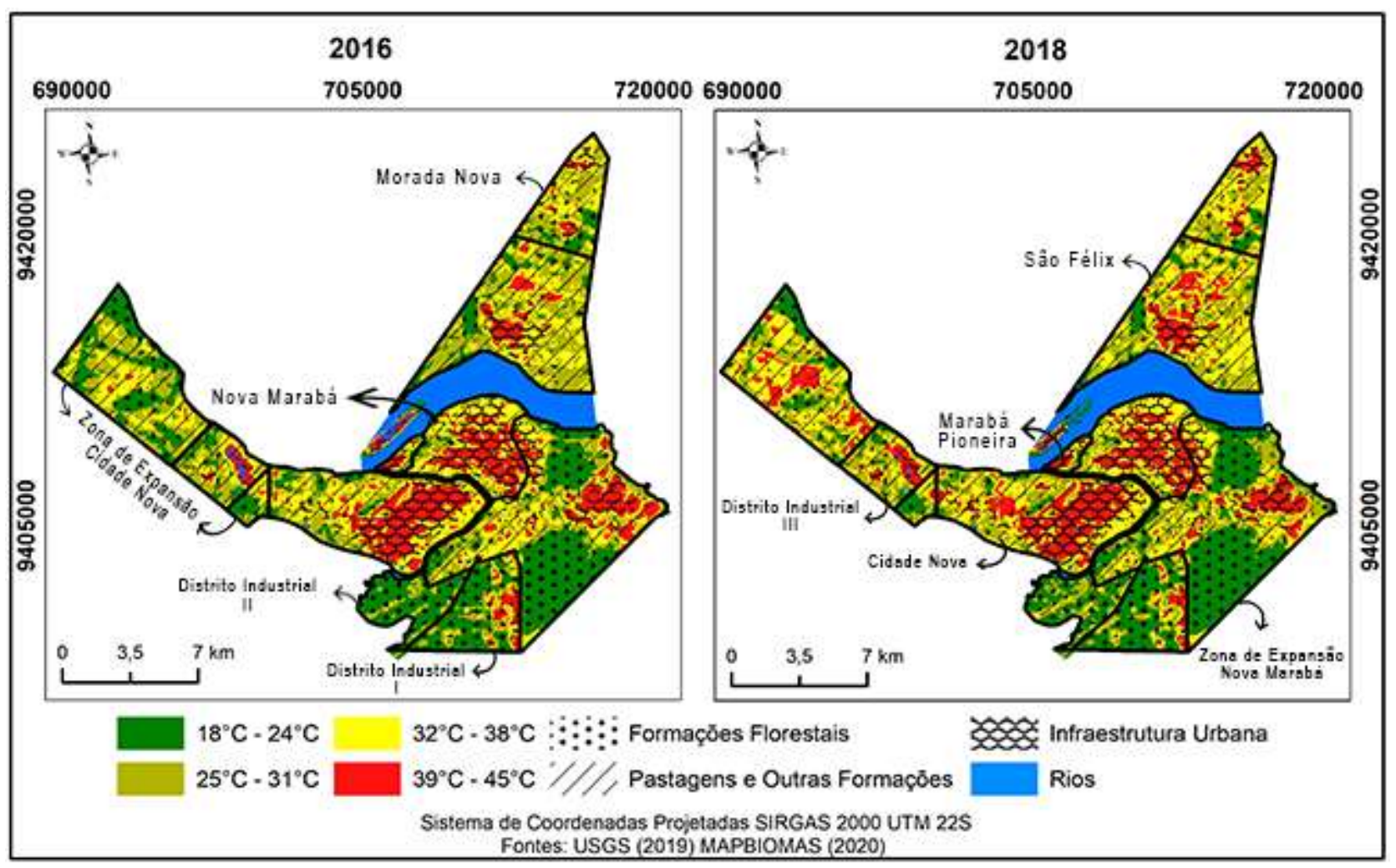

Fonte: Autores (2020).

A utilização do solo de Marabá estava dividida de forma que as áreas de Formações Florestais representavam 124,07 km² em 2016 e 126,88 km² em 2018, já as áreas de Pastagens e Outra Formações correspondiam 101,28km² em 2016 e 107,77 
$\mathrm{km}^{2}$ em 2018, por último como mostra a Figura 5, o uso destinado a Infraestrutura Urbana tinha uma área de 41,51 $\mathrm{km}^{2} \mathrm{em}$ 2016 e $45,92 \mathrm{~km}^{2}$ em 2018.

Caracterizado por um aumento das temperaturas mínimas e máximas em comparação à análise anterior, o ano de 2016 em relação a TST consolida as formações de aglomerações caloríferas nos núcleos Cidade Nova, Nova Marabá, Zonas de Expansão Nova Marabá e Distrito Industrial III; onde as áreas de Pastagens correspondem às temperaturas na escala de $33,36^{\circ} \mathrm{C}$ a $35,75^{\circ} \mathrm{C}$ e as de Infraestrutura Urbana com valores climáticos de $35,75^{\circ} \mathrm{C}$ chegando a $42,16^{\circ} \mathrm{C}$, esses maiores valores encontrados em áreas com edificações e desenvolvimento vertical, resultado semelhante alcançado no estudo de Barboza et al. (2020).

A dinâmica de utilização do solo marabaense em 2016 representou de maneira significativa alguns fatores que acarretaram para os resultados de temperatura encontrados, que foi o aumento de áreas de Infraestrutura Urbana como a construção e inauguração de mais um empreendimento do PMCMV (Residencial Jardim do Éden no Núcleo Morada Nova) constituído como a primeira iniciativa de produção habitacional deste núcleo, antes predominada por ocupações irregulares (Lima; Leão, 2015).

Em comparação ao ano de 1988 que em trinta anos a temperatura superficial terrestre mínima da cidade de Marabá teve um aumento de $6,9^{\circ} \mathrm{C}$, enquanto as temperaturas máximas da superfície elevou-se em $11,73^{\circ} \mathrm{C}$.

Confirmando as formações de ilhas de calor na cidade de Marabá, os mapas espaço- temporais mostram que em todos os núcleos urbanos houveram a formação desse fenômeno climático e a consolidação das ilhas caloríferas naqueles com maior habitação e densidade urbanística como os núcleos Cidade Nova e Nova Marabá, observou-se também que os núcleos São Félix e Zona de Expansão Nova Marabá, foram os que tiveram maiores formações de novas ilhas de calor ao longo dos anos.

Constatou-se que a área caracterizada como Formações Florestais sofreu uma diminuição de 33,73 km², resultado esse que infere nos resultados de TST encontrados e nos de outros usos do solo. Nesta perspectiva e em face dos dados citados, as áreas de Pastagens e Outras Formações, Infraestrutura Urbana aumentaram 25,91 km² e 23,68 km² respectivamente.

A Figura 6 mostra o comportamento da Temperatura Superficial Terrestre (TST) obtidos no trabalho em Marabá em 30 anos (1988-2018), de maneira que é possível notar uma diminuição dos valores de temperatura máxima $\left({ }^{\circ} \mathrm{C}\right)$ nos anos de 1996 e 2001, seguidos de uma elevação contínua dos valores mínimos e máximos de temperatura. 
Figura 6: Evolução da Temperatura Superficial Terrestre (TST) e dados de Precipitação Total Diária (mm) e Evaporação do Piche Diária (mm) para a cidade de Marabá.

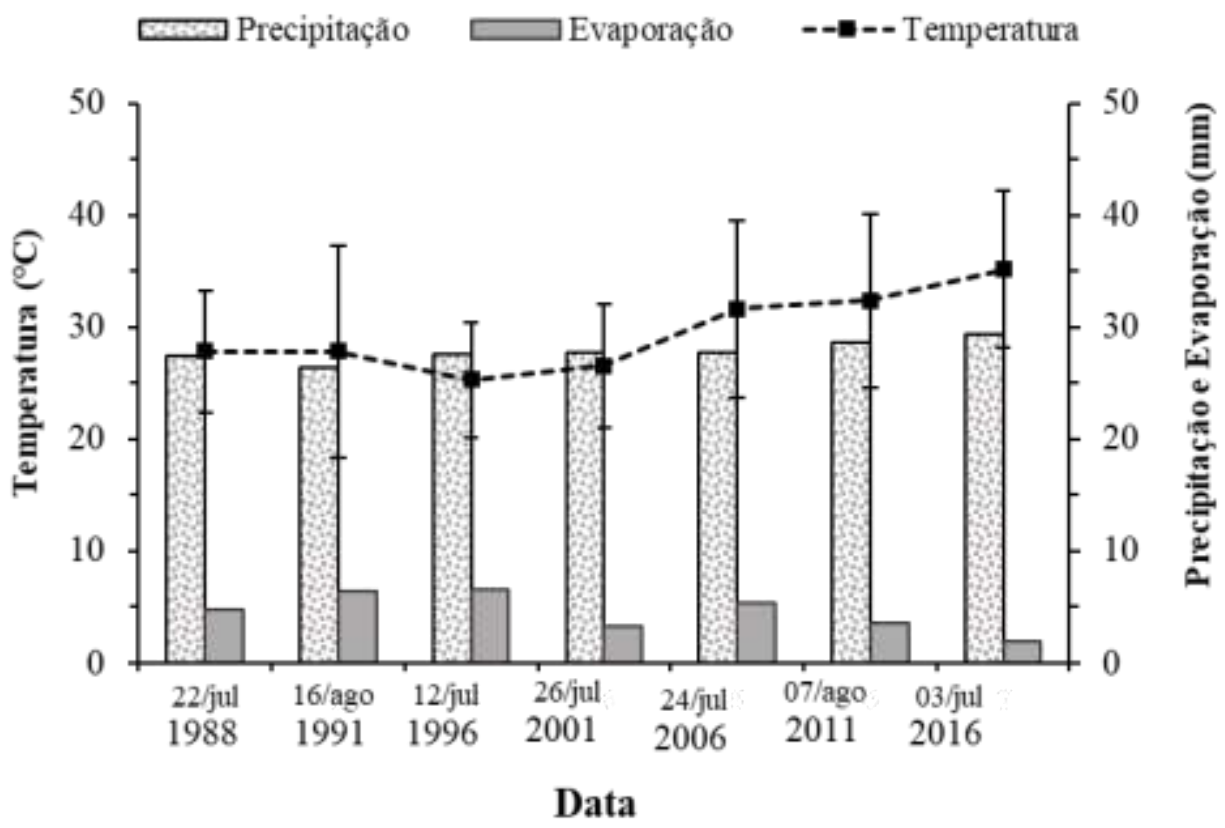

Fonte: Autores (2020).

É possível relacionar essa informação com o fato de os anos de 1991 e 1996 serem os anos com maiores valores de Formações Florestais em comparação aos outros anos, frisando o vínculo entre a dinâmica de superfície com o desempenho climático da região.

Além disso, a Figura 6 apresenta os dados de Precipitação Total Diária $(\mathrm{mm})$ e Evapotranspiração (Evaporação do Piche Diária) (mm) das mesmas datas das imagens de satélite de onde foram obtidos os valores de temperatura (INMET, 2019), fatores esses que se interligam diretamente com a temperatura e possuem influência em ambos os resultados encontrados.

Fortemente influenciada pelo tipo de vegetação por meio da evapotranspiração das plantas, a Evaporação é um fator climático intrínseco ao uso e ocupação do solo e seus resultados observados na Figura 6 podem ser relacionados ao comportamento da dinâmica terrestre. Variando entre valores de 6,5 (1996) e 1,9 (2016), observa-se uma diminuição desses valores de acordo com o tempo, paralelo a redução de áreas vegetadas verificadas nos mapas anteriormente.

Os dados de Precipitação Total Diária (mm) refletem a estação climática da época de coleta dos mesmos; considerado como verão amazônico, o período menos chuvoso na região norte acontece no período do inverno (junho a setembro), fator esse que pode estar relacioando os valores encontrados variando de 26,4 (1991) a 29,38 (2016).

O ano de 2018 não se insere na relação Temperatura e Precipitação Total Diária (mm) e Evaporação do Piche Diária $(\mathrm{mm})$ visto que o INMET disponibiliza valores nulos para esse ano, logo não foi viável a utilização dessas informações.

No que diz respeito aos valores de temperatura mínima, o ano de 1991 apresentou a menor quantificação com 18,38 ${ }^{\circ} \mathrm{C}$ e o ano de 2018 com o maior valor $29,27^{\circ} \mathrm{C}$; os valores máximos de temperatura tiveram em 1996 a sua menor captação com $30,42^{\circ} \mathrm{C}$ e em 2018 o maior valor, com $44,99^{\circ} \mathrm{C}$. Esse aumento nos valores de temperatura acompanha e se relaciona com a diminuição das áreas de Formações Florestais em face ao aumento das áreas de Pastagens e Infraestrutura Urbana nos anos observados. 


\section{Conclusão}

Em relação a distribuição espacial das ilhas de calor no perímetro urbano de Marabá, verificou-se a formação e consolidação das mesmas principalmente nos núcleos Nova Marabá e Cidade Nova, sendo esses os que possuem uma maior área de edificações e ocupação urbana em relação aos outros núcleos. Foi observado que os núcleos Morada Nova, São Félix e Zona de Expansão Nova Marabá foram os que tiveram maior formação de novas ilhas de calor a partir do ano de 2011, influenciado principalmente pelos projetos de habitação instalados naqueles núcleos em anos anteriores.

Ao analisar as informações adquiridas a partir dos mapas temáticos, observou-se o aumento de 35,26\% dos valores de temperatura máxima em detrimento da diminuição de 43,82\% das áreas de Formações Florestais.

Por fim analisa-se que os resultados climáticos encontrados em Marabá são inerentes a toda e qualquer cidade de médio porte do Brasil, como a expansão da malha urbana desenfreada e sem planejamento, redução de áreas vegetadas, com elevadas temperaturas, eventos extremos de precipitação e menor evaporação, fatores esses que podem contribuir para diminuição da umidade dos ambientes e a ocorrência de problemas de saúde e stress térmico.

Sugere-se a realização de estudos posteriores sobre os impactos socio-ambientais citados anteriormente, de forma a buscar possíveis soluções e melhorar o arrefecimento das cidades, reiterando sempre a importância dos estudos do clima para o planejamento urbano e melhoria da qualidade ambiental dos centros urbanos.

\section{Referências}

Almeida Jr., W. F., Simões, P. P. S., \& Morais, R. C. S. (2019). Análise da temperatura de superfície de área descampada no município de Teresina-PI com a utilização de sensoriamento remoto para os anos de 2007 e 2017. In: Congresso Brasileiro de Gestão Ambiental, 10. 2019. Anais... Fortaleza

Ayouade, J. O. (1998). Introdução à Climatologia para os trópicos. (5a ed.), Bertland Brasil, 332p.

Barboza, E. N., Bezerra Neto, F. das C., \& Caiana, C. R. A. (2020). Sensoriamento Remoto aplicado à análise do fenômeno de Ilhas de Calor Urbano na cidade de Vitória, Espírito Santo. Research, Society and Development. 9(6), e187963655, 10.33448/rsd-v9i6.3655.

Barboza, E. N., Bezerra Neto, F. das C., \& Caiana, C. R. A. (2020) Geoprocessamento aplicado na análise dos efeitos da urbanização no campo térmico em Fortaleza, Ceará. Research, Society and Development. 9(7), e57973731. 10.33448/rsd-v9i7.3731.

Barsi, J. A., Barker, J. L., \& Schott, J. R. (2003). An Atmospheric Correction Parameter Calculator for a Single Thermal Band Earth-Sensing Instrument. IGARSS03, p. 21- 25 July 2003, Centre de Congres Pierre Baudis, Toulouse, France. http://atmcorr.gsfc.nasa.gov/Barsi_IGARSS03.PDF

Barsi, J. A., Schott, J. R., Palluconi, F. D., \& Hook, S. J. (2005). Validation of a web-based atmospheric correction tool for single thermal band instruments. Proceedings of SPIE, 5882. http://dx.doi.org/10.1117/12.619990.

Borges, V. V., \& Zaidan, R. T. (2011). Levantamento Do Diferencial Térmico Da Superfície Da Bacia Hidrográfica Do Rio Paraibuna- MG/RJ/2008, Pelo Sensor Termal Do Satélite LANDSAT-5. Revista de Geografia-PPGEO-UFJF, 1(2).

Cremonez, F.E., Cremonez, P. A., Feroldi, M., Camargo, M. P., Klajn, F. F., \& Feiden, A. (2014). Avaliação de impacto ambiental: metodologias aplicadas no Brasil. Revista Monografias Ambientais, 13(5), 3821-3830.

Environmental System Research Institute - ESRI (2017). ArcGis Professional GIS for desktop, version 10.5.

Fernandes, L. A. (2015). Ambientes urbanos e fatores naturais na conformação das condições climáticas no período de inverno em Viçosa/MG.. Revista de Ciências Humanas, Viçosa, 15(2), 366-380.

Gartland, L. (2010). Ilhas de Calor: como mitigar zonas de calor em áreas urbanas. Oficina do Texto.

Instituto Brasileiro De Geografia E Estatística - IBGE. (2019). Malha geométrica dos municípios brasileiros. Rio de Janeiro: Coordenação de Estruturas Territoriais, a partir do Arquivo Gráfico Municipal - AGM. http://dados.gov.br/dataset/malha-geometrica-dos-municipios-brasileiros.

Instituto Nacional De Meteorologia - INMET. Banco de Dados Meteorológicos do INMET 1986 a 2016. 2019. https://bdmep.inmet.gov.br/.

Köppen, W., \& Geiger, R. (1928). Klimate der Erde. Gotha: Verlag Justus Perthes. Wall- map 150cmx200cm.

Lima, J. J. F., \& Leão, R. F. C. (2015). O Programa Minha Casa, Minha Vida E A Expansão Urbana Na Cidade De Marabá-PA. In: Encontro Nacional da Associação Nacional de Pós-Graduação e Pesquisa em Planejamento Urbano e Regional, 16. 2015. Anais... Belo Horizonte.

Lopes, J. S. G., Aleixo, N. C. R., \& Da Silva Neto, J. C. A. (2019). Amplitude Térmica E Magnitude Das Ilhas De Calor Em Tefé-AM, Brasil. Boletim de Geografia, 37(1), 251-264. 
Research, Society and Development, v. 10, n. 7, e41710716718, 2021

(CC BY 4.0) | ISSN 2525-3409 | DOI: http://dx.doi.org/10.33448/rsd-v10i7.16718

Lucena, A. J. (2012) A Ilha de calor na região metropolitana do Rio de Janeiro. 2012. 473 p. Dissertação (Doutorado em Engenharia Civil) . COPPE/Universidade Federal do Rio de Janeiro, Rio de Janeiro - RJ.

Marabá. Lei Municipal n ${ }^{17}$ 17846, de 29 de março de 2018. Dispõe sobre a revisão do Plano Diretor Participativo do município de Marabá, instituído pela Lei Municipal $n^{\circ} 17.213$ de 09 de outubro de 2006, e dá outras providências. Marabá, PA. 2018. http://www.governotransparente.com.br/transparencia/documentos/4466490/download/ 29/Plano_Diretor_Participativo_\%2017.846_Mar\%C3\%A7o_2018.pdf

Marengo, J.A., Tomasella, J., Soares, W. R., Alves, L. M., \& Nobre, C. A. (2012). Extreme climatic events in the Amazon basin. Theoretical Applied Climatology, 107, 73-85.

Nascimento, D. T. F., \& Oliveira, I. J. (2011). Análise Da Evolução Do Fenômeno De Ilhas De Calor No Município De Goiânia-GO (1986-2010). Boletim goiano de geografia, 31(2), 113-127.

Nichol, J. E. A. (1994) GIS-based approach to microclimate monitoring in Singapore's high- rise housing estates. Photogrammetric Engineering \& Remote Sensing, 60(10), $1225-1232$.

Oscar Jr, A. C. S., \& Brandão, A. M. P. M. (2015). O Sistema Clima Urbano da Ilha do Fundão (RJ): Subsídio para o Planejamento Urbano. Revista Brasileira de Geografia Física, 8(1), 113-127.

Pires, E. G., \& Ferreira Jr, L. G. Mapeamento da temperatura de superfície a partir de imagens termais dos satélites Landsat 7 e Landsat 8 . (2015). In: Simpósio Brasileiro De Sensoriamento Remoto-SBSR, 17. 2015. Anais... João Pessoa, 7421-7428.

PRIMAVESI, O; ARZABE, C; SANTOS, M.(2007). Mudanças climáticas: visão tropical integrada das causas, dos impactos e de possíveis soluções para ambientes rurais ou urbanos. São Carlos, Brasil: Embrapa Pecuária Sudeste.

PROJETO MAPBIOMAS. Coleção 4.1 da Série Anual de Mapas de Cobertura e Uso de Solo do Brasil. https://mapbiomas.org/colecoes-mapbiomas1?cama_set_language=pt-BR.

Qin, Z.; Karnieli A., \& Berliner, P. (2001). A mono-window algorithm for retrieving land surface temperature from Landsat TM data and its application to the Israel-Egypt border region. International Journal of Remote Sensing, 22(18), 3719-3746.

Ramos, M. F., Ribeiro, C. B. M., \& Andrade, M. P. (2016). Estudo Da Variação Temporal Do Índice De Vegetação Por Diferença Normalizada Em Juiz De Fora A Partir De Imagens De Satélite. Revista de Geografia- PPGEO-UFJF, 6(1), 13-21.

Silva, E. M. da, Barboza, E. N., Morais, J. M. P. de, Souza, J. H. A. de, \& Oliveira, B. B. de. (2020). Análise de sensação térmica no município de Barbalha, Ceará. Research, Society and Development. 9(7), p. e98973795. 10.33448/rsd-v9i7.3795.

Sousa, S. B.,\& Ferreira Jr, L. G. (2012) Relação entre temperatura de superfície terrestre, índices espectrais e classes de cobertura da terra no município de Goiânia (GO), Raega, 26, 75-99.

Souza Filho, J. D. C., Ribeiro, A., Costa, M. H, Cohen, J. C. P., \& Rocha, E. J. P. (2006). Variação sazonal do balanço de radiação em uma floresta tropical no nordeste da Amazônia. Revista Brasileira de Meteorologia, 21(3b), 318-330.

Sposito, M. E. B., Elias, D., Soares, B. R. (2016). Agentes econômicos e reestruturação urbana e regional: Marabá e Los Ángeles. Editora Cultura Acadêmica.

United States Geological Survey - USGS. Imagem do satélite Landsat. United States: Earth Explorer, 2019. https://earthexplorer.usgs.gov/.

Velho, L. F. (2014). Análise Da Dinâmica Da Temperatura De Superfície E Da Ocupação Urbana No Município De Porto Alegre. Dissertação (Doutorado em Sensoriamento Remoto). Universidade Federal do Rio Grande do Sul. 